\title{
Prospective study of alcohol consumption and the incidence of the metabolic syndrome in US men
}

\author{
Mark Stoutenberg ${ }^{1 *}$, Duck-chul Lee ${ }^{2}$, Xuemei Sui ${ }^{3}$, Steven Hooker ${ }^{4}$, Viviana Horigian ${ }^{1}$, \\ Tatiana Perrino ${ }^{1}$ and Steven Blair ${ }^{3,5}$ \\ ${ }^{1}$ Department of Epidemiology and Public Health, University of Miami, Miller School of Medicine, \\ 1120 Northwest 14th Street, Suite 1014, Miami, FL 33136, USA \\ ${ }^{2}$ Department of Kinesiology, Iowa State University, Ames, IA, USA \\ ${ }^{3}$ Department of Exercise Science, Arnold School of Public Health, University of South Carolina, Columbia, SC, USA \\ ${ }^{4}$ Exercise and Wellness Program, School of Nutrition and Health Promotion, Arizona State University, Tempe, AZ, USA \\ ${ }^{5}$ Department of Epidemiology and Biostatistics, Arnold School of Public Health, University of South Carolina, \\ Columbia, SC, USA \\ (Submitted 27 April 2012 - Final revision received 14 November 2012 - Accepted 27 November 2012 - First published online 23 January 2013)
}

\begin{abstract}
Few studies have evaluated the effects of alcohol consumption on the incidence of the metabolic syndrome (MetS). Therefore, the objective of the present study was to examine the association between alcohol consumption and incident MetS in a population of US men. This is a prospective study of 7483 Caucasian men, who were free of the MetS and CVD at baseline. Information was collected on alcohol consumption, health status and fitness level at an initial clinical examination. Additional health information and determination of incident cases of the MetS were obtained at follow-up clinical examinations between 1979 and 2005. Compared with non-drinkers, the multivariate hazard ratios of the MetS for light (1-3 drinks/week), moderate (4-7 drinks/week), moderate-heavy ( $8-13$ drinks/week) and heavy ( $\geq 14$ drinks/week) drinkers were 0.81 (95\% CI $0.68,0.95), 0.68$ (95\% CI $0.57,0.80), 0.70$ (95\% CI $0.59,0.83)$ and 0.78 (95\% CI $0.66,0.91)$, respectively. This association was seen across age groups, in men with one or more pre-existing MetS risk factors, and those with BMI $\geq 25 \mathrm{~kg} / \mathrm{m}^{2}$, and in all alcohol beverage types at most levels of alcohol consumption. An inverse dose-response association between alcohol consumption and low HDL concentrations was observed, while significant associations were observed between high fasting glucose concentrations and moderate, moderate-heavy and heavy levels of alcohol consumption. Alcohol consumption was not significantly associated with central obesity, hypertriacylglycerolaemia or hypertension. All levels of alcohol consumption provided significant inverse associations with incidence of the MetS. In particular, this effect was observed in overweight and/or obese individuals, in those who had pre-existing risk factors for the MetS, and extended across all types of alcoholic beverages consumed.
\end{abstract}

Key words: Alcohol: Metabolic syndrome: CVD risk factors: Prospective studies

The metabolic syndrome (MetS) is a constellation of metabolic abnormalities that includes central obesity, low HDL concentrations, high fasting glucose and TAG concentrations, and elevated blood pressure (BP), which is fast becoming a major public health challenge ${ }^{(1)}$. This combination of cardiometabolic risk factors has received an increasing level of attention due to its strong association with the development of diabetes and increased risk of cardiovascular morbidity and mortality ${ }^{(2,3)}$. According to Ford et al. ${ }^{(4)}$, the age-adjusted prevalence of the MetS in the US population is $23 \%$, yet many of the mechanisms underlying its development remain unknown. Given the prevalence of the MetS, there is increasing importance to obtain a greater understanding of the modifiable factors that may mitigate or moderate the progression of events leading towards its development.

Among these modifiable factors, there has only recently been interest in examining the effect of alcohol consumption on the development of the MetS. Alcohol consumption is an accepted part of the American culture, as an estimated $76 \%$ of American men engage in alcohol consumption over the course of a year, with $33 \%$ of men reporting alcohol consumption on any given day $^{(5)}$. Previous studies have established strong evidence for a reduced risk of $\mathrm{CHD}^{(6)}$, stroke ${ }^{(7)}$ and mortality ${ }^{(8)}$ with light-tomoderate levels of alcohol consumption, with heavier levels of consumption resulting in negative health consequences. Moderate-to-high levels of alcohol consumption have been

Abbreviations: BP, blood pressure; HDL-C, HDL-cholesterol; HR, hazard ratio; MetS, metabolic syndrome.

*Corresponding author: Professor M. Stoutenberg, fax +1 305243 3651, email mstoutenberg@med.miami.edu 
associated with hypertension, liver disease, peptic ulcers, certain types of cancers, pregnancy complications and brain damage $^{(9)}$.

Given the popularity of alcohol and its diverse effects on health and chronic disease, it is important to obtain a clearer understanding of its association with the MetS. The extent of this association is controversial as the existing literature is filled with conflicting findings. A recent meta-analysis concluded that light-to-moderate drinking provides a beneficial metabolic effect ${ }^{(10)}$. However, individual studies have described the relationship between alcohol consumption and the MetS as being J-shaped $^{(11)}$, U-shaped ${ }^{(12)}$, an inverse relationship ${ }^{(13,14)}$, a linear relationship ${ }^{(15,16)}$ or having no relationship at all ${ }^{(17,18)}$. Yet, only a handful of these studies have prospectively examined alcohol consumption and the incidence of the MetS ${ }^{(16-20)}$, and, of these, only two have been conducted in North America ${ }^{(16,21)}$. Contributing to this controversy, there appear to be sex differences as have been found by investigators: the prevalence of the MetS to be higher in women than in men who abstained from drinking ${ }^{(13)}$; a positive association of alcohol consumption in women only ${ }^{(18)}$; a similar effect of moderate alcohol consumption in both sexes ${ }^{(11)}$; no association in either sex ${ }^{(17)}$. A final weakness in previous studies involves the adjustment for physical activity levels. Studies examining the relationship between cardiorespiratory fitness and the MetS have identified an inverse association ${ }^{(22)}$, whereas a study using self-reported physical activity exposure was equivocal $^{(23)}$. Cardiorespiratory fitness is a stronger predictor of health than self-reported physical activity, and may therefore serve as a more accurate modifying variable ${ }^{(24)}$.

Therefore, the purpose of the present study was to examine the prospective association between alcohol consumption and the incidence of the MetS in a population of US men, and to determine whether this relationship is modified by cardiorespiratory fitness, BMI, age and baseline health status.

\section{Experimental methods}

\section{Study population}

Participants were 20-100-year-old men (mean 43.4 (SD 9.0) years) who had preventive medical examinations at the Cooper Clinic (Dallas, TX, USA) between 1979 and 2005 and who were enrolled in the Aerobics Center Longitudinal Study. Criteria for inclusion in the present analysis required individuals to have had at least two clinical examinations with complete measurements for each MetS variable and a baseline cardiorespiratory fitness assessment ( $n$ 12551). Individuals were excluded based on pre-existing cases of the MetS ( $n$ 2419), CVD (including heart attack or stroke), cancer or an abnormal resting or exercise electrocardiogram $(n$ 1282) at baseline. In addition, those not achieving $\geq 85 \%$ of age-predicted maximal heart rate on the treadmill test at baseline ( $n 88)$ or who had less than 1 year of follow-up ( $n$ 1279) were excluded. The remaining 7483 men met all criteria for the present study and were included in the final analysis. The study was conducted according to the guidelines laid down in the Declaration of Helsinki, and all procedures involving human subjects/patients were approved by the Cooper Institute Institutional Review Board. Written informed consent was obtained from all subjects/ patients for the baseline clinical examination and follow-up study.

\section{Definition of the metabolic syndrome}

The outcome variable of the MetS was defined, following the guidelines of the National Cholesterol Education Program Adult Treatment Panel III (NCEP ATP III), as meeting three or more of the following criteria: abdominal obesity (waist circumference $>102 \mathrm{~cm}$ ); TAG $\geq 15 \mathrm{mg} /$; HDL-cholesterol (HDL-C) $<4 \mathrm{mg} / \mathrm{l}$; resting systolic or diastolic BP of $\geq 130$ or $\geq 85 \mathrm{mmHg}$, respectively; a fasting blood glucose $\geq 10 \mathrm{mg}$ / $\mathrm{l}^{(1)}$. Self-reported history of physician-diagnosed hypertension and diabetes was included in the definition of abnormal BP and glucose, respectively, as done in other epidemiological studies investigating the incidence of the $\operatorname{MetS}^{(21)}$.

\section{Alcohol exposure}

At their initial clinical visit, as part of the Personal Habits section of their Medical History Questionnaire, individuals were asked a series of ten questions regarding their alcohol use, specifically whether they drank alcoholic beverages and how many drinks per week they consumed of beer, wine and hard liquor. Alcohol consumption was quantified as the number of drinks per week, with a drink standardised to 12 ounces $(360 \mathrm{ml})$ of beer, 5 ounces $(150 \mathrm{ml})$ of wine or 1.5 ounces $(22.5 \mathrm{ml})$ of liquor. Individuals were then classified into five categories of alcohol consumption (non-drinkers: 0 drinks; light: 1-3 drinks/week; moderate: 4-7 drinks/week; moderateheavy: 8-13 drinks/week; heavy: $14+$ drinks/week), with those who reported no recent alcohol consumption (including both former drinkers and abstainers) serving as the reference group. These classifications are in accordance with current USDA Dietary Guidelines ${ }^{(1)}$ that defines moderate alcohol consumption in men as two drinks per $\mathrm{d}$ and heavy or high-risk drinkers consuming more than four drinks per $d$ or fourteen drinks per week. Since few individuals limited their drinking to only one type of alcohol, individuals were categorised as a beer, wine or liquor drinker if they reported consuming any of these alcohol beverages.

\section{Clinical examination}

The clinical examination followed a $12 \mathrm{~h}$ fast as described previously ${ }^{(25,26)}$. Briefly, participants completed a medical questionnaire consisting of demographic questions, lifestyle habits, and past and present chronic disease history. In addition, they underwent a clinical evaluation that included body composition assessment, blood chemistry analysis, BP measurement and a physical examination by a physician. Waist circumference was taken at the level of the umbilicus with an inelastic tape and classified as $\leq 102.0$ or $>102.0 \mathrm{~cm}$ according to clinical guidelines ${ }^{(27)}$. Resting BP was measured by standard auscultatory methods after at least 5 min of seated rest and was recorded as the average of two or more readings separated by $2 \min ^{(28)}$. 
Concentrations of TAG, HDL-C and glucose were measured from the blood obtained from the antecubital venous blood and serum was analysed using automated bioassays in the Cooper Clinic Laboratory according to the Centers for Disease Control and Prevention's Lipid Standardization Program standards $^{(28)}$. BMI was calculated from measured weight and height, and individuals were categorised as normal weight $\left(18.5-24.9 \mathrm{~kg} / \mathrm{m}^{2}\right)$, overweight $\left(25.0-29.9 \mathrm{~kg} / \mathrm{m}^{2}\right)$ and obese $\left(\geq 30 \mathrm{~kg} / \mathrm{m}^{2}\right)^{(27)}$. Smoking status and a personal history of hypertension, diabetes, heart attack, stroke and cancer were ascertained through a standardised medical questionnaire. Individuals were also stratified according to the number of MetS risk factors present at baseline to allow for the examination of alcohol consumption in groups with and without co-morbidities.

\section{Cardiorespiratory fitness}

Participants completed a maximal exercise treadmill test to assess their cardiorespiratory fitness. Cardiorespiratory fitness was quantified as the duration of the maximal treadmill exercise test using a modified Balke protocol ${ }^{(25)}$. Participants were encouraged to reach their maximal effort and those who did not achieve $85 \%$ of their age-predicted maximal heart rate were excluded, as it was assumed that they probably had subclinical medical problems and less than near-maximal effort would lead to an underestimation of fitness. The duration of this treadmill test is highly correlated with measured maximal oxygen uptake in men $\left(\begin{array}{ll}r & 0.92\end{array}\right)^{(29)}$. Classification of fitness level was determined on the basis of fifths of treadmill time, with those having fitness levels in the lowest $20 \%$ classified as the 'unfit' and the remainder classified as the 'fit'. This cut point was used as there is no consensus for the clinical definition of being 'unfit' and because it has been used as a similar guideline in previous Aerobics Center Longitudinal Study investigations, which have shown low fitness to be an independent risk factor for various morbidity and mortality outcomes ${ }^{(30,31)}$.

\section{Statistical analyses}

Baseline characteristics were summarised across the levels of alcohol consumption and tests for linear trends were calculated using general linear models. MetS cases were defined as individuals who met the MetS definition at any clinical examination after baseline. The follow-up time was computed as the difference between the date of the baseline examination and the first follow-up event of the MetS or the last clinical examination through 2005. A Cox proportional hazards model was used to estimate the hazard ratios (HR) and 95\% CI of the MetS across the categories of alcohol consumption. Ordinal linear trends across the five categories of alcohol consumption were tested using Cox regression models. In Cox regression models, baseline age, examination year, current smoking status and total treadmill time were considered as potential confounders as in previous studies ${ }^{(21,31,32)}$. The proportional hazards assumption was tested by examining the log-log survival plots grouped on exposure categories.

Further multivariate analyses were conducted based on stratification by age $(<55$ or $\geq 55$ years), BMI $(<25$ or $\geq 25 \mathrm{~kg} / \mathrm{m}^{2}$ ), number of pre-existing MetS risk factors (0, 1 or 2) and fitness level (fit or unfit). In the multivariate analysis for type of alcoholic beverage (beer, wine and liquor), there were insufficient numbers of drinkers who consumed a single beverage type exclusively. Therefore, this analysis was conducted separately for all individuals who consumed each type of alcoholic beverage while adjusting for the remaining types of alcohol consumed. Finally, the association between alcohol consumption and the individual components of the MetS was evaluated. To calculate multivariate HR, Cox proportional hazards models containing each of the components of the MetS as binary-dependent variables were fitted to the same data which were utilised for the association between alcohol consumption and the incidence of the MetS. The SAS (SAS 9.1; SAS Institute, Inc.) program was used to conduct statistical analyses. All $P$ values were two-sided, and $P<0.05$ was considered as statistically significant.

\section{Results}

From 1979 to 2005, 7483 eligible men were followed for an average of 6.0 (SD 5.2) years for a total of 44943 personyears of follow-up. Descriptive characteristics of the study participants are presented in Table 1. Significant differences in baseline characteristics were observed between men in the different categories of alcohol consumption except for abdominal obesity. In general, heavier drinkers were more likely to be older, current smokers, and have a higher BMI, systolic and diastolic BP, fasting glucose concentrations, total cholesterol, HDL-C and TAG concentrations.

During an average of 6.0 years of follow-up, 1578 new cases of the MetS were observed. Overall, the incidence rate of the MetS was 35.1 per 1000 person-years. The HR of the MetS across the five categories of alcohol consumption levels are presented in Table 2. Significant linear trends were observed across the five categories of alcohol consumption $(P<0.002)$. In model 1 , with adjustment for age, year of examination and smoking, all levels of alcohol consumption showed a significant inverse association with the incidence of the MetS compared with non-drinkers. After additional adjustment for fitness level (model 2), all associations between the levels of alcohol consumption and the incidence of the MetS remained significant.

Table 3 presents the HR of the individual components of the MetS across the different categories of alcohol consumption. No effect of alcohol consumption was observed on central obesity, high TAG concentrations or hypertension. Moderate (28\%), moderate-heavy (22\%) and heavy (56\%) levels of drinking were associated with a higher risk of high fasting glucose concentrations. A significant positive association was observed between HDL-C and all levels of alcohol consumption in a dose-response manner with greater levels of alcohol consumption associated with a lower risk of low HDL-C.

In Table 4, the HR of the MetS across the five categories of alcohol consumption are presented after stratification by age, BMI, pre-existing MetS components, fitness level and type of alcohol beverage consumed. Among individuals less than 55 years of age, inverse associations between alcohol 
Table 1. Baseline characteristics of the sample population (Mean values and standard deviations)

\begin{tabular}{|c|c|c|c|c|c|c|c|c|c|c|c|}
\hline & \multicolumn{11}{|c|}{ Alcohol consumption (drinks/week) } \\
\hline & \multicolumn{2}{|c|}{ Non-drinkers } & \multicolumn{2}{|c|}{ C1 $(1-3)$} & \multicolumn{2}{|c|}{ C2 (4-7) } & \multicolumn{2}{|c|}{ C3 $(8-13)$} & \multicolumn{2}{|c|}{$\mathrm{C} 4(14+)$} & \multirow[b]{2}{*}{$P$ for linear trend } \\
\hline & Mean & SD & Mean & SD & Mean & SD & Mean & SD & Mean & SD & \\
\hline Subjects $(n)$ & \multicolumn{2}{|c|}{870} & \multicolumn{2}{|c|}{1541} & \multicolumn{2}{|c|}{1795} & \multicolumn{2}{|c|}{1509} & \multicolumn{2}{|c|}{1768} & \\
\hline Age (years) & $44 \cdot 64$ & 9.46 & $42 \cdot 34$ & 9.05 & 43.31 & 8.97 & 43.36 & 8.77 & 44.02 & 8.85 & $<0.001$ \\
\hline BMl $\left(\mathrm{kg} / \mathrm{m}^{2}\right)$ & $25 \cdot 01$ & 2.96 & $25 \cdot 26$ & $2 \cdot 85$ & $25 \cdot 29$ & $2 \cdot 61$ & $25 \cdot 28$ & $2 \cdot 65$ & $25 \cdot 40$ & 2.47 & 0.017 \\
\hline Alcohol consumption (drinks/week) & 0 & & 2.05 & 0.77 & 5.38 & $1 \cdot 12$ & $10 \cdot 23$ & 1.61 & 24.39 & 14.48 & $<0.001$ \\
\hline Maximal treadmill time (min) & $19 \cdot 48$ & 4.38 & $20 \cdot 23$ & 4.41 & $20 \cdot 28$ & 4.34 & $20 \cdot 33$ & $4 \cdot 30$ & $20 \cdot 15$ & 4.34 & $<0.001$ \\
\hline Waist circumference (cm) & $90 \cdot 12$ & 8.58 & 89.70 & 8.65 & $90 \cdot 11$ & 7.96 & $90 \cdot 35$ & $7 \cdot 77$ & $90 \cdot 89$ & 7.76 & $<0.001$ \\
\hline Systolic blood pressure (mmHg) & $117 \cdot 71$ & $12 \cdot 19$ & 117.54 & 11.40 & 117.57 & $12 \cdot 00$ & $118 \cdot 20$ & $11 \cdot 71$ & $119 \cdot 23$ & $12 \cdot 25$ & $<0.001$ \\
\hline Diastolic blood pressure $(\mathrm{mmHg})$ & 78.93 & $8 \cdot 77$ & 78.53 & 8.63 & $78 \cdot 81$ & 8.91 & $79 \cdot 22$ & 8.33 & 79.97 & 8.57 & $<0.001$ \\
\hline FBG $(\mathrm{mg} / \mathrm{l})$ & $953 \cdot 3$ & 113 & 959.8 & $120 \cdot 8$ & 965.9 & $103 \cdot 1$ & 977 & 103.9 & 987.9 & $105 \cdot 3$ & $<0.001$ \\
\hline HDL-C (mg/l) & $449 \cdot 3$ & $103 \cdot 3$ & $467 \cdot 7$ & 110.9 & $489 \cdot 2$ & 109.9 & $509 \cdot 1$ & $116 \cdot 1$ & 529.5 & 120 & $<0.001$ \\
\hline TAG $(\mathrm{mg} / \mathrm{l})$ & $1047 \cdot 6$ & $617 \cdot 2$ & $1037 \cdot 7$ & $599 \cdot 3$ & $1028 \cdot 7$ & 514 & $1047 \cdot 4$ & $566 \cdot 3$ & $1096 \cdot 5$ & $581 \cdot 3$ & 0.005 \\
\hline Total cholesterol (mg/l) & $2030 \cdot 1$ & $362 \cdot 2$ & 1997.3 & 371.6 & $2032 \cdot 6$ & 362.9 & 2057.5 & $366 \cdot 3$ & $2137 \cdot 6$ & $386 \cdot 8$ & $<0.001$ \\
\hline Current smokers (\%) & \multicolumn{2}{|c|}{$7 \cdot 70$} & \multicolumn{2}{|c|}{11.94} & \multicolumn{2}{|c|}{$13 \cdot 20$} & \multicolumn{2}{|c|}{$17 \cdot 56$} & \multicolumn{2}{|c|}{$21 \cdot 66$} & $<0.001$ \\
\hline \multicolumn{12}{|l|}{ MetS risk factors (\%) } \\
\hline Abdominal obesity* & \multicolumn{2}{|c|}{$6 \cdot 32$} & \multicolumn{2}{|c|}{5.91} & \multicolumn{2}{|c|}{5.46} & \multicolumn{2}{|c|}{$5 \cdot 30$} & \multicolumn{2}{|c|}{5.43} & 0.819 \\
\hline High blood pressure & \multirow{2}{*}{\multicolumn{2}{|c|}{30.57}} & \multicolumn{2}{|c|}{$26 \cdot 80$} & \multicolumn{2}{|c|}{$29 \cdot 19$} & \multicolumn{2}{|c|}{$30 \cdot 48$} & \multicolumn{2}{|c|}{33.65} & $<0.001$ \\
\hline High FBG $\ddagger$ & \multirow{2}{*}{\multicolumn{2}{|c|}{$\begin{array}{l}24.48 \\
32.41\end{array}$}} & \multicolumn{2}{|c|}{$26 \cdot 61$} & \multicolumn{2}{|c|}{$32 \cdot 03$} & \multicolumn{2}{|c|}{$35 \cdot 72$} & & & $<0.001$ \\
\hline Low HDL-C§ & & & & & & & & & & & $<0.001$ \\
\hline High TAG\| & & & & & & & & & & & 0.051 \\
\hline
\end{tabular}

C, category; FBG, fasting blood glucose; MetS, metabolic syndrome; HDL-C, HDL-cholesterol.

Defined as a waist circumference $>102 \mathrm{~cm}$.

† Defined as a systolic blood pressure $\geq 130 \mathrm{mmHg}$, a diastolic blood pressure $\geq 85 \mathrm{mmHg}$ or a history of physician-diagnosed hypertension.

$\ddagger$ Defined as a $F B G \geq 10 \mathrm{mg} / \mathrm{l}$ or a history of physician-diagnosed diabetes.

$\S$ Defined as an $\mathrm{HDL}$ concentration $<4 \mathrm{mg} /$.

Defined as TAG concentrations $\geq 15 \mathrm{mg} / \mathrm{l}$ 
Table 2. Hazard ratios of the metabolic syndrome across the levels of alcohol consumption

(Hazard ratios and $95 \%$ confidence intervals)

\begin{tabular}{|c|c|c|c|c|c|c|c|c|c|}
\hline & \multicolumn{9}{|c|}{ Alcohol consumption (drinks/week) } \\
\hline & \multirow[b]{2}{*}{ Non-drinkers } & \multicolumn{2}{|c|}{$\mathrm{C} 1(1-3)$} & \multicolumn{2}{|c|}{ C2 (4-7) } & \multicolumn{2}{|c|}{ C3 $(8-13)$} & \multicolumn{2}{|c|}{$\mathrm{C} 4(14+)$} \\
\hline & & Hazard ratio & $95 \% \mathrm{Cl}$ & Hazard ratio & $95 \% \mathrm{Cl}$ & Hazard ratio & $95 \% \mathrm{Cl}$ & Hazard ratio & $95 \% \mathrm{Cl}$ \\
\hline$n$ & 870 & \multicolumn{2}{|c|}{1541} & \multicolumn{2}{|c|}{1795} & \multicolumn{2}{|c|}{1509} & \multicolumn{2}{|c|}{1768} \\
\hline No. of cases & 248 & \multicolumn{2}{|c|}{322} & \multicolumn{2}{|c|}{333} & \multicolumn{2}{|c|}{298} & \multicolumn{2}{|c|}{377} \\
\hline Model $1^{*}$ & 1.00 (reference) & 0.78 & $0.66,0.93$ & 0.64 & $0.54,0.75$ & 0.66 & $0.55,0.78$ & 0.73 & $0.62,0.86$ \\
\hline Model $2 \dagger$ & 1.00 (reference) & 0.81 & $0.68,0.95$ & 0.68 & $0.57,0.80$ & 0.70 & $0.59,0.83$ & 0.78 & $0.66,0.91$ \\
\hline
\end{tabular}

C, category.

${ }^{*}$ Adjusted for age (in years), year of examination, smoking (current smoker or not).

† Additionally adjusted for maximal treadmill time ( $\mathrm{min})$.

consumption and the risk of the MetS were observed with all categories except light consumption, while similar inverse associations were seen in the light, moderate and moderate-heavy categories in men 55 years of age and older. Among individuals with a BMI $\geq 25 \mathrm{~kg} / \mathrm{m}^{2}$, all levels of alcohol consumption resulted in a lower incidence of the MetS; however, among those with a BMI $<25 \mathrm{~kg} / \mathrm{m}^{2}$, a positive association was seen only among moderate consumers. A separate sub-analysis (not shown) was conducted for individuals with BMI $\geq 30 \mathrm{~kg} / \mathrm{m}^{2}$, but results were inconclusive due to the small number of cases in this category. Moderate, moderate-heavy and heavy alcohol consumption all resulted in an inverse association in individuals who had two pre-existing components for the MetS, while no significant associations were seen in men with no pre-existing MetS components at baseline. Significant inverse associations were observed in both fit and unfit individuals in the moderate, moderateheavy and heavy alcohol consumption categories. Table 4 also presents the HR of the MetS across the five levels of alcohol consumption after stratification by type of alcohol beverage. Across each type of alcoholic beverage, there were similar significant inverse associations, except with heavy levels of liquor consumption.

\section{Discussion}

In the present prospective study of US men, we observed that all levels of alcohol consumption provided a significant inverse association with the development of the MetS after taking into account several potential confounding factors. In particular, the present study showed a significantly lower risk of the MetS in individuals who had a greater number of MetS risk factors present at baseline and were overweight/ obese. The benefits of alcohol also remained significant regardless of the type of alcoholic beverage consumed.

The main finding of the present study that alcohol consumption was associated with a lower risk of MetS incidence expands upon previous epidemiological studies indicating that light-to-moderate levels of alcohol consumption are associated with a lower risk of the MetS in $\operatorname{men}^{(10,14,33,34)}$. However, few studies examining the relationship between alcohol consumption and the MetS have been conducted in prospective studies $^{(16,19,20)}$, and only one ${ }^{(16)}$ has shown that moderate-to-heavy alcohol consumption is associated with a significantly lower risk of the MetS, as seen in the present study. Although this inverse association with alcohol consumption was seen in moderate drinkers, the present data also indicate that these benefits also extend to moderateheavy and heavy levels of alcohol consumption. However, these results must be interpreted with caution as these findings do not take into account the numerous health hazards associated with heavy alcohol use (e.g. gastrointestinal and liver diseases, cancer, intentional injuries, etc. ${ }^{(35)}$ ).

There are many reasons for the varying results observed when examining the association between alcohol consumption and the MetS, including factors such as sex and race/ ethnicity $^{(20)}$. There are several racial and ethnic biological differences that influence alcohol metabolism ${ }^{(36)}$, as well as racial/ethnic variations in drinking quantities, patterns and alcohol abuse disorders ${ }^{(37)}$. In contrast to the present study, Baik \& Shin ${ }^{(19)}$ found a linear trend between alcohol consumption and the incidence of the MetS, with the quartile of heaviest drinkers displaying a $63 \%$ increase in relative risk. However, this cohort consisted of Korean men and women who have different alcohol metabolism rates ${ }^{(38)}$. Therefore, appropriate consideration must be given to race, sex and ethnicity when making comparisons between existing studies.

Another major confounding factor is the definition of the MetS itself. Wakabayashi ${ }^{(12)}$ found no significant associations between alcohol consumption and the prevalence of the MetS using International Diabetes Federation guidelines ${ }^{(39)}$, but did find a significant association in heavy drinkers when using the National Cholesterol Education Program - Adult Treatment Panel III guidelines ${ }^{(1)}$. Other factors, such as quantification of drinking patterns ${ }^{(40)}$, frequency ${ }^{(41)}$ and lifetime alcohol consumption ${ }^{(15)}$, may also have a differential impact on the development of the MetS, yet are rarely assessed and/or controlled for in a standardised manner when examining this relationship.

\section{Effect of alcohol on individual metabolic syndrome components}

In addition to the direct effect that alcohol consumption has on the development of the MetS, there may be an indirect effect through a series of relationships with each individual 
component of the MetS. Understanding these individual relationships may be of particular use when individualising guidance for patients who may be at risk for some components of the MetS and not others. In the present study, we observed no increase in the risk of hypertension with any level of alcohol consumption. This result is consistent with other prospective reports that have indicated no increase in the incidence of hypertension with light-to-moderate levels of alcohol consumption in white men ${ }^{(42,43)}$. However, these prospective studies, as well as other cross-sectional studies, have shown a higher risk of hypertension with moderate-toheavy levels of alcohol consumption in comparison with non-drinkers ${ }^{(12,19,33)}$. In the present study, there was no observed effect of alcohol consumption on either central obesity or serum TAG concentrations. Several studies, in agreement with the present findings, have demonstrated that alcohol consumption is not associated with increased waist circumference $^{(12,14,33)}$. A limited number of investigators have demonstrated an increased risk of moderate and heavy drinking on serum TAG concentrations ${ }^{(12,19)}$. In contrast, the present results suggest that no observed level of alcohol consumption has a negative impact on serum TAG, a finding supported by others ${ }^{(14)}$. Although alcohol consumption has been associated with decreased serum glucose concentrations, particularly in light and moderate drinkers ${ }^{(14)}$, the present results indicate that moderate, moderate-heavy and heavy levels of drinking are associated with an increased risk of hyperglycaemia, which agrees with a recent study showing increased risk in heavy drinkers ${ }^{(19)}$. Paradoxically, alcohol consumption has been related to enhanced insulin sensitivity and lower plasma insulin concentrations ${ }^{(44,45)}$, providing a possible explanation for the lower incidence of type 2 diabetes in alcohol consumers compared with abstainers ${ }^{(16,46)}$. One area of consistent agreement involves the present findings of a positive dose-response relationship between alcohol consumption and the risk of low HDL-C, which is in agreement with others $^{(12,19,44)}$. Thus, it appears that the possible beneficial effect of alcohol consumption is that it is associated with a lower risk of low HDL-C, and may offset the deleterious effect of an increased risk of high fasting glucose concentrations. It appears that there is a need for further studies to clarify the physiological mechanisms and relationship between alcohol consumption and the individual components of the MetS.

\section{Stratification based on BMI, age, fitness level and baseline health status}

All levels of alcohol consumption, except light consumers, resulted in a reduced risk of the MetS in individuals $<55$ years. In individuals $\geq 55$ years of age, this association was seen in light, moderate and moderate-heavy drinkers. These results appear to indicate that moderate and moderate-heavy alcohol consumption have a positive impact on the incidence of the MetS across all ages in men. There was a lower risk of MetS incidence in men who consumed moderate, moderate-heavy and heavy levels of alcohol and who were positive for two MetS components at baseline. However, in men with no pre-existing MetS components at 
Table 4. Hazard ratios of the metabolic syndrome across the levels of alcohol consumption after stratification by age, BMI, pre-existing metabolic syndrome (MetS) components, fitness level and beverage type*

(Hazard ratios and $95 \%$ confidence intervals)

\begin{tabular}{|c|c|c|c|c|c|c|c|c|c|c|c|}
\hline & \multirow[b]{3}{*}{ Cases/total } & \multicolumn{10}{|c|}{ Alcohol consumption (drinks/week) } \\
\hline & & \multirow[b]{2}{*}{ Non-drinkers } & \multicolumn{2}{|c|}{$\mathrm{C} 1(1-3)$} & \multicolumn{2}{|c|}{ C2 (4-7) } & \multicolumn{2}{|c|}{ C3 $(8-13)$} & \multicolumn{2}{|c|}{$\mathrm{C} 4(14+)$} & \multirow[b]{2}{*}{$P$ for interaction } \\
\hline & & & Hazard ratio & $95 \% \mathrm{Cl}$ & Hazard ratio & $95 \% \mathrm{Cl}$ & Hazard ratio & $95 \% \mathrm{Cl}$ & Hazard ratio & $95 \% \mathrm{Cl}$ & \\
\hline Age (years) & & & & & & & & & & & 0.72 \\
\hline$<55$ & $1394 / 6615$ & 1.00 (reference) & 0.86 & $0.72,1.03$ & 0.70 & $0.58,0.83$ & 0.72 & $0.60,0.86$ & 0.74 & $0.62,0.89$ & \\
\hline$\geq 55$ & $184 / 868$ & 1.00 (reference) & 0.50 & $0.30,0.84$ & 0.55 & $0.35,0.87$ & 0.60 & $0.37,0.95$ & 0.69 & $0.45,1.06$ & \\
\hline BMI $\left(\mathrm{kg} / \mathrm{m}^{2}\right)$ & & & & & & & & & & & $0 \cdot 16$ \\
\hline$<25$ & $532 / 3705$ & 1.00 (reference) & 1.04 & $0.78,1.38$ & 0.65 & $0.48,0.88$ & 0.81 & $0.61,1.09$ & 0.89 & $0.67,1.18$ & \\
\hline$\geq 25$ & $1046 / 3778$ & 1.00 (reference) & 0.72 & $0.58,0.89$ & 0.66 & $0.54,0.81$ & 0.64 & $0.52,0.79$ & 0.62 & $0.51,0.76$ & \\
\hline $\begin{array}{c}\text { No. of pre-existing MetS } \\
\text { components } \dagger\end{array}$ & & & & & & & & & & & 0.45 \\
\hline 0 & $197 / 2237$ & 1.00 (reference) & 0.96 & $0.60,1.54$ & 0.66 & $0.41,1.07$ & 0.70 & $0.43,1.13$ & 0.92 & $0.58,1.47$ & \\
\hline 1 & $543 / 2926$ & 1.00 (reference) & 0.70 & $0.52,0.94$ & 0.78 & $0.59,1.03$ & 0.74 & $0.55,0.99$ & 0.67 & $0.51,0.90$ & \\
\hline 2 & $838 / 2320$ & 1.00 (reference) & 0.88 & $0 \cdot 70,1 \cdot 10$ & 0.62 & $0.49,0.78$ & 0.70 & $0.55,0.88$ & 0.75 & $0.60,0.93$ & \\
\hline Fitness level $\ddagger$ & & & & & & & & & & & 0.66 \\
\hline Fit & $1119 / 5978$ & 1.00 (reference) & 0.85 & $0.69,1.04$ & 0.65 & $0.53,0.80$ & 0.69 & $0.56,0.84$ & 0.71 & $0.58,0.86$ & \\
\hline Unfit & $459 / 1505$ & 1.00 (reference) & 0.81 & $0.59,1 \cdot 10$ & 0.71 & $0.53,0.96$ & 0.68 & $0.50,0.94$ & 0.74 & $0.55,0.99$ & \\
\hline Alcohol beverage§ & & & & & & & & & & & NS \\
\hline Beer & $1167 / 5569$ & 1.00 (reference) & 0.75 & $0.62,0.91$ & 0.75 & $0.63,0.90$ & 0.66 & $0.54,0.81$ & 0.72 & $0.59,0.88$ & \\
\hline Wine & $1103 / 5217$ & 1.00 (reference) & 0.67 & $0.55,0.82$ & 0.67 & $0.55,0.82$ & 0.74 & $0.61,0.90$ & 0.71 & $0.58,0.86$ & \\
\hline Liquor & $1020 / 4487$ & 1.00 (reference) & 0.63 & $0.50,0.78$ & 0.72 & $0.60,0.88$ & 0.73 & $0.60,0.90$ & 0.87 & $0.71,1.06$ & \\
\hline
\end{tabular}

C, category.
${ }^{*}$ All stratified variables were adjusted for age (in years), year of examination, smoking (current smoker or not), number of pre-existing MetS components and maximal treadmill time (min), except variables within their same level of *All stratified variables were adjusted for age (in years), year of examination, smoking (current smoker or not), number of pre-existing MetS components and maximal treadmill time (min), except variables within their same level of
stratification.
† Defined as any of the following conditions: systolic/diastolic blood pressure $\geq 140 / 90 \mathrm{mmHg}$ or history of hypertension; fasting glucose $\geq 12.6 \mathrm{mg} / \mathrm{l}$ or history of diabetes; dyslipidaemia (total cholesterol $\geq 24 \mathrm{mg} / / \mathrm{l}, \mathrm{TAG} \geq 20 \mathrm{mg} / \mathrm{l}$ or † Defined as any of the following conditions: systolic/diastolic blood pressure $\geq 140 / 90 \mathrm{mmHg}$ or history of hypertension; fasting guco
HDL-cholesterol $<4 \mathrm{mg} / \mathrm{l})$.

HDL-cholesterol $<4 \mathrm{mg} / /)$.
$\ddagger$ Defined as the most fit $(80 \%)$ and the least fit $(20 \%)$ based on the distribution of maximal treadmill times from the study cohort.
$\S$ Alcohol beverage stratification was created based on the distribution of use of each alcohol type with all remaining types of alcohol included as covariates in the model. No interaction test was performed between the type of conol consumption. 
baseline (i.e. those who might be considered healthier), there was no lower risk with any level of alcohol consumption. This result is consistent with others who found the relationship between alcohol consumption and the MetS the strongest among individuals with a higher number of MetS components ${ }^{(33)}$ and in men with an increased risk of diabetes ${ }^{(44)}$. A lower risk of the MetS was also seen in individuals with a BMI $\geq 25 \mathrm{~kg} / \mathrm{m}^{2}$ at all levels of alcohol consumption. This finding is in discord with others who observed that heavy drinking in overweight individuals was associated with a $37-115 \%$ increase in the risk of the MetS ${ }^{(19,34)}$. Finally, all levels of alcohol consumption, except for light drinkers, were associated with a lower risk of MetS incidence in both the fit and unfit men in the study. Previous literature has established a significant inverse relationship between cardiorespiratory fitness and the MetS in similar cohorts ${ }^{(21,22)}$. However, the mechanisms behind the lower risk of the MetS in unfit individuals with higher levels of alcohol consumption are unclear, and additional work is needed to further clarify this association.

\section{Beverage-specific effects on the metabolic syndrome}

Across each type of alcoholic beverage, similar lower risks of the MetS were observed within each category of alcohol consumption, except with high levels of liquor consumption. The present results support those of Djousse et al. ${ }^{(33)}$ who found that consumption of wine only, beer only, spirits only and any combination of beverage type was associated with risk reductions in the MetS of 68, 58, 43 and 44\%, respectively. These findings are in contrast to those reporting that heavy liquor consumption results in a significant increase in the MetS ${ }^{(19)}$, and that beer and wine consumption, but not liquor, resulted in a lower prevalence of the $\mathrm{MetS}^{(14)}$. The present finding is of particular interest given that previous studies have primarily demonstrated that the beneficial effect of alcohol consumption has been limited to wine drinkers, individuals who are more likely to engage in healthier lifestyles ${ }^{(47)}$.

\section{Strengths and limitations}

The strengths of the present study include the use of a comprehensive physical examination and an extensive follow-up period in one of the largest cohort studies to investigate the incidence of the MetS by alcohol consumption, as well as our ability to stratify and adjust our models using cardiorespiratory fitness rather than self-reported physical activity. Furthermore, this is one of the first prospective studies examining the relationship between alcohol consumption and the MetS in a US male population, enhancing the internal validity of the present findings. The limitations of the present study include: limited external validity beyond Caucasian males of a higher socio-economic status; possible under-reporting and subsequent misclassification of alcohol consumption; the potential misclassification of the reference group as former drinkers and lifetime abstainers have very different alcohol consumption histories. Additionally, in the stratified analyses, the relatively small number of incident MetS cases in the older, normal-weight
(BMI $<25 \mathrm{~kg} / \mathrm{m}^{2}$ ), healthy and least fit groups may have affected the results in these groups.

\section{Conclusion}

The results of the present study suggest that alcohol consumption may protect against the development of the MetS. These findings may be particularly useful for health professionals in providing individualised guidance to their patients with regard to alcohol consumption and its effect on the MetS or its individual components, and in assisting with the future establishment of guidelines regarding the benefits and risks of alcohol consumption.

\section{Acknowledgements}

We thank the Cooper Clinic physicians and technicians for collecting the baseline data, and staff at the Cooper Institute for the data entry and data management. The present study was supported by the National Institutes of Health grants (AG06945, HL62508 and DK088195), and an unrestricted research grant from The Coca-Cola Company. The content is solely the responsibility of the authors and does not necessarily represent the official views of the National Institutes of Health. The authors' contributions are as follows: S. B. designed the research; S. B. and S. H. conducted the research; D.-C. L. analysed the data; X. S., V. H., T. P. and M. S. wrote the paper; M. S. and S. B. had primary responsibility for the final content. All authors read and approved the final manuscript. None of the authors has any conflict of interest to disclose.

\section{References}

1. Anonymous (2002) Third Report of the National Cholesterol Education Program (NCEP) Expert Panel on Detection, Evaluation, and Treatment of High Blood Cholesterol in Adults (Adult Treatment Panel III) Final Report no. 106. Baltimore, MD: Lippincott Williams \& Wilkins.

2. Alberti KG, Eckel RH, Grundy SM, et al. (2009) Harmonizing the metabolic syndrome: a joint interim statement of the International Diabetes Federation Task Force on Epidemiology and Prevention; National Heart, Lung, and Blood Institute; American Heart Association; World Heart Federation; International Atherosclerosis Society; and International Association for the Study of Obesity. Circulation 120, $1640-1645$.

3. Mottillo S, Filion KB, Genest J, et al. (2010) The metabolic syndrome and cardiovascular risk a systematic review and meta-analysis. J Am Coll Cardiol 56, 1113-1132.

4. Ford ES, Giles WH \& Dietz WH (2002) Prevalence of the metabolic syndrome among US adults: findings from the third National Health and Nutrition Examination Survey. JAMA 287, 356-359.

5. Guenther PM, Bowman SA, Goldman JD (2010) Alcoholic Beverage Consumption by Adults 21 Years and Over in the United States: Results from the National Health and Nutrition Examination Survey, 2003-2006: Technical Report. Center for Nutrition Policy and Promotion, and Agricultural Research Service, US Department of Agriculture.

6. Hvidtfeldt UA, Tolstrup JS, Jakobsen MU, et al. (2010) Alcohol intake and risk of coronary heart disease in younger, middle-aged, and older adults. Circulation 121, 1589-1597. 
7. Sacco RL, Elkind M, Boden-Albala B, et al. (1999) The protective effect of moderate alcohol consumption on ischemic stroke. JAMA 281, 53-60.

8. Di Castelnuovo A, Costanzo S, Bagnardi V, et al. (2006) Alcohol dosing and total mortality in men and women: an updated meta-analysis of 34 prospective studies. Arch Intern Med 166, 2437-2445.

9. US Department of Health \& Human Services and US Department of Agriculture (HHS/USDA) (2010) Dietary Guidelines for Americans, 2010. Washington, DC: US Government Printing Office.

10. Alkerwi A, Boutsen M, Vaillant M, et al. (2009) Alcohol consumption and the prevalence of metabolic syndrome: a meta-analysis of observational studies. Atherosclerosis 204, 624-635.

11. Yoon YS, Oh SW, Baik HW, et al. (2004) Alcohol consumption and the metabolic syndrome in Korean adults: the 1998 Korean National Health and Nutrition Examination Survey. Am J Clin Nutr 80, 217-224.

12. Wakabayashi I (2011) Association between alcohol drinking and metabolic syndrome in Japanese male workers with diabetes mellitus. J Atheroscler Thromb 18, 684-692.

13. Park YW, Zhu S, Palaniappan L, et al. (2003) The metabolic syndrome: prevalence and associated risk factor findings in the US population from the Third National Health and Nutrition Examination Survey, 1988-1994. Arch Intern Med 163, 427-436.

14. Freiberg MS, Cabral HJ, Heeren TC, et al. (2004) Alcohol consumption and the prevalence of the metabolic syndrome in the US: a cross-sectional analysis of data from the Third National Health and Nutrition Examination Survey. Diabetes Care 27, 2954-2959.

15. Fan AZ, Russell M, Naimi T, et al. (2008) Patterns of alcohol consumption and the metabolic syndrome. J Clin Endocrinol Metab 93, 3833-3838.

16. Gigleux I, Gagnon J, St-Pierre A, et al. (2006) Moderate alcohol consumption is more cardioprotective in men with the metabolic syndrome. J Nutr 136, 3027-3032.

17. Buja A, Scafato E, Sergi G, et al. (2010) Alcohol consumption and metabolic syndrome in the elderly: results from the Italian longitudinal study on aging. Eur J Clin Nutr 64, 297-307.

18. Wilsgaard T \& Jacobsen BK (2007) Lifestyle factors and incident metabolic syndrome. The Tromso Study 1979-2001. Diabetes Res Clin Pract 78, 217-224.

19. Baik I \& Shin C (2008) Prospective study of alcohol consumption and metabolic syndrome. Am J Clin Nutr 87, $1455-1463$.

20. Carnethon MR, Loria CM, Hill JO, et al. (2004) Risk factors for the metabolic syndrome: the Coronary Artery Risk Development in Young Adults (CARDIA) study, 1985-2001. Diabetes Care 27, 2707-2715.

21. Carnethon MR, Gidding SS, Nehgme R, et al. (2003) Cardiorespiratory fitness in young adulthood and the development of cardiovascular disease risk factors. JAMA $\mathbf{2 9 0}$, 3092-3100.

22. LaMonte MJ, Barlow CE, Jurca R, et al. (2005) Cardiorespiratory fitness is inversely associated with the incidence of metabolic syndrome: a prospective study of men and women. Circulation 112, 505-512.

23. Laaksonen DE, Lakka HM, Salonen JT, et al. (2002) Low levels of leisure-time physical activity and cardiorespiratory fitness predict development of the metabolic syndrome. Diabetes Care 25, 1612-1618.

24. Lee DC, Sui X, Ortega FB, et al. (2011) Comparisons of leisure-time physical activity and cardiorespiratory fitness as predictors of all-cause mortality in men and women. $\mathrm{Br}$ J Sports Med 45, 504-510.

25. Blair SN, Kohl HW 3rd, Paffenbarger RS Jr, et al. (1989) Physical fitness and all-cause mortality. A prospective study of healthy men and women. JAMA 262, 2395-2401.

26. Blair SN, Kampert JB, Kohl HW, et al. (1996) Influences of cardiorespiratory fitness and other precursors on cardiovascular disease and all-cause mortality in men and women. JAMA 276, 205-210.

27. Expert Panel on the Identification, Evaluation, and Treatment of Overweight and Obesity in Adults (1998) Executive summary of the clinical guidelines on the identification, evaluation, and treatment of overweight and obesity in adults. Arch Intern Med 158, 1855-1867.

28. Pickering TG, Hall JE, Appel LJ, et al. (2005) Recommendations for blood pressure measurement in humans and experimental animals: part 1: blood pressure measurement in humans: a statement for professionals from the Subcommittee of Professional and Public Education of the American Heart Association Council on High Blood Pressure Research. Hypertension 45, 142-161.

29. Pollock ML, Bohannon RL, Cooper KH, et al. (1976) A comparative analysis of four protocols for maximal treadmill stress testing. Am Heart J 92, 39-46.

30. Sui X, LaMonte MJ, Laditka JN, et al. (2007) Cardiorespiratory fitness and adiposity as mortality predictors in older adults. JAMA 298, 2507-2516.

31. Lee DC, Sui X, Church TS, et al. (2009) Associations of cardiorespiratory fitness and obesity with risks of impaired fasting glucose and type 2 diabetes in men. Diabetes Care 32, $257-262$.

32. Sui X, Hooker SP, Lee IM, et al. (2008) A prospective study of cardiorespiratory fitness and risk of type 2 diabetes in women. Diabetes Care 31, 550-555.

33. Djousse L, Arnett DK, Eckfeldt JH, et al. (2004) Alcohol consumption and metabolic syndrome: does the type of beverage matter? Obes Res 12, 1375-1385.

34. Zhu S, St-Onge MP, Heshka S, et al. (2004) Lifestyle behaviors associated with lower risk of having the metabolic syndrome. Metabolism 53, 1503-1511.

35. Room R, Babor $\mathrm{T} \&$ Rehm J (2005) Alcohol and public health. Lancet 365, 519-530.

36. National Institute on Alcohol Abuse and Alcoholism (2004) Unpublished Data from the 2001-2002 National Epidemiologic Survey on Alcohol and Related Conditions (NESARC), a Nationwide Survey of 43,093 U.S. Adults Aged 18 Or Older.

37. Chartier K \& Caetano R (2010) Ethnicity and health disparities in alcohol research. Alcohol Res Health 33, 152-160.

38. Agarwal DP, Harada S \& Goedde HW (1981) Racial differences in biological sensitivity to ethanol: the role of alcohol dehydrogenase and aldehyde dehydrogenase isozymes. Alcohol Clin Exp Res 5, 12-16.

39. Alberti KG, Zimmet P, Shaw J, et al. (2005) The metabolic syndrome - a new worldwide definition. Lancet 366, 1059-1062.

40. Lee KW, Park BJ, Kang HT, et al. (2011) Alcohol-drinking patterns and metabolic syndrome risk: the 2007 Korean National Health and Nutrition Examination Survey. Alcohol 45, 499-505.

41. Mukamal KJ, Jensen MK, Gronbaek M, et al. (2005) Drinking frequency, mediating biomarkers, and risk of myocardial infarction in women and men. Circulation $\mathbf{1 1 2}$ 1406-1413. 
42. Fuchs FD, Chambless LE, Whelton PK, et al. (2001) Alcohol consumption and the incidence of hypertension: The Atherosclerosis Risk in Communities Study. Hypertension 37, $1242-1250$.

43. Sesso HD, Cook NR, Buring JE, et al. (2008) Alcohol consumption and the risk of hypertension in women and men. Hypertension 51, 1080-1087.

44. Wannamethee SG, Shaper AG, Perry IJ, et al. (2002) Alcohol consumption and the incidence of type II diabetes. J Epidemiol Commun Health 56, 542-548.
45. Facchini F, Chen YD \& Reaven GM (1994) Light-to-moderate alcohol intake is associated with enhanced insulin sensitivity. Diabetes Care 17, 115-119.

46. Koppes LL, Dekker JM, Hendriks HF, et al. (2005) Moderate alcohol consumption lowers the risk of type 2 diabetes: a meta-analysis of prospective observational studies. Diabetes Care 28, 719-725.

47. Rosell M, De Faire U \& Hellenius ML (2003) Low prevalence of the metabolic syndrome in wine drinkers - is it the alcohol beverage or the lifestyle? Eur J Clin Nutr 57, 227-234. 